\title{
Crack-free Micromachining of Glass Ceramic Using Visible LIBWE
}

\author{
Ji-Yen Cheng ${ }^{* 1,2,3,4}$, Huai-Yi Chen ${ }^{1,5,6}$, Mansoureh Z. Mousavi ${ }^{1,6,7}$, Chou Y.Y. Chang ${ }^{1}$ \\ ${ }^{* 1}$ Research Center for Applied Sciences, Academia Sinica Taiwan, Taipei City, 11529, Taiwan ROT \\ ${ }^{* 2}$ Institute of Biophotonics, National Yang-Ming University, Taipei City 11221, Taiwan \\ ${ }^{* 3}$ Molecular Imaging Research Center, National Yang-Ming University, Taipei City 11221, Taiwan \\ ${ }^{* 4}$ Department of Mechanical and Mechatronic Engineering, National Taiwan Ocean University, \\ Keelung 20224, Taiwan \\ ${ }^{* 5}$ Department of Engineering and System Science, National Tsing-Hua University, Taiwan \\ ${ }^{*}$ Nano Science and Technology Program, Taiwan International Graduate Program, Academia \\ Sinica Taiwan \\ ${ }^{* 7}$ Department of Chemistry, National Taiwan University, Taiwan
}

\begin{abstract}
Zerodur is a glass ceramic that has zero thermal expansion coefficient. It has good optical transmission in the visible light range. The zero thermal expansion property of Zerodur makes it an important substrate material for precision optical applications such as astronomical telescope and EUVL (13 nm) lithography. In this work, we demonstrated the crack-free micromachining of Zerodur using visible laser-induced-backside-wet-etching (v-LIBWE). We used eutectic Indium Gallium alloy $(\mathrm{In} / \mathrm{Ga})$ as the light absorber for the $\mathrm{v}$-LIBWE process. Spectra of the light emission during the ablation or etching were recorded and compared using the real-time monitoring method. Measuring the intensity of the characteristic emission peaks allow us to determine the etching threshold in a more efficient way.
\end{abstract}

DOI: $10.2961 / \mathrm{j} 1 \mathrm{mn} .2013 .03 .0011$

Keywords: visible LIBWE, v-LIBWE, Zerodur, glass machining, laser etching, liquid-assisted, direct-writing, nanosecond

\section{Introduction}

Zerodur is a glass ceramic that has extraordinary property of zero thermal expansion coefficient between $-50{ }^{\circ} \mathrm{C}$ to $100{ }^{\circ} \mathrm{C}\left(0 \pm 0.10 \times 10^{-6} \mathrm{~K}^{-1}\right.$ for class 2 product $)[1]$. Zerodur has good optical transmission in the visible light range (Transmission $>80 \%$ from $460 \mathrm{~nm}$ to $2500 \mathrm{~nm}$ ). The composition (in $\mathrm{wt} \%$ ) of Zerodur is $57.2 \mathrm{SiO}_{2}, 25.3 \mathrm{Al}_{2} \mathrm{O}_{3}$, 6.5 $\mathrm{P}_{2} \mathrm{O}_{5}, 3.4 \mathrm{Li}_{2} \mathrm{O}, 1.0 \mathrm{MgO}, 1.4 \mathrm{ZnO}, 0.2 \mathrm{Na}_{2} \mathrm{O}, 0.4 \mathrm{~K}_{2} \mathrm{O}$, $0.5 \mathrm{As}_{2} \mathrm{O}_{3}, 2.3 \mathrm{TiO}_{2}$, and $1.8 \mathrm{ZrO}_{2}$ [1]. Compared to other common optical glass (BK7, thermal expansion coefficient $=7.1 \times 10^{-6} \mathrm{~K}^{-1}$; fused silica, thermal expansion coefficient $=0.54 \times 10^{-6} \mathrm{~K}^{-1}$ ), the zero thermal expansion property of Zerodur makes it an important substrate material for precision optical applications such as astronomical telescope and EUVL (13 nm) lithography.

Zerodur can be structured using CNC machining [2] but the reports on the micromachining of Zerodur are relatively few. Especially, the use of laser on the micromachining is rarely reported.

Laser-induced-backside-wet-etching (LIBWE) is a technique for micromachining of transparent substrate using excimer laser (wavelength $\lambda=248 \mathrm{~nm}$ ) or diodepumped solid state (DPSS) lasers $(\lambda=266 \mathrm{~nm}$ and $351 \mathrm{~nm}$ ) [3-7]. The LIBWE process is used to etch transparent material with high precision and to produce near optical quality surface. The advantages of LIBWE include lower etching threshold (1 order smaller than direct ablation for fusedsilica [5]), higher effectiveness for transparent material, and smaller heat-affected zone (HAZ) when comparing to the traditional direct laser ablation.
Recently LIBWE using visible laser, i.e. v-LIBWE, has been demonstrated using nanosecond frequency doubled DPSS lasers $(\lambda=532 \mathrm{~nm}$, pulse duration $\sim 10 \mathrm{~ns})$ together with organic dye absorbers[8-10] or metallic absorbers $[11,12]$. The v-LIBWE uses economical green laser to obtain surface quality that is identical to that by traditional UV LIBWE. In addition, optical alignment using visible light is more convenient than using UV light. According to previous research [13], the LIBWE using liquid metallic absorbers has higher etching rate and threshold fluence than those by using hydrocarbon absorbers.

Because Zerodur is not transparent in UV range, using v-LIBWE instead of UV LIBWE is a more practical approach for laser direct-writing micromachining. In this work, we used nanosecond $532 \mathrm{~nm}$ laser and metallic absorber, eutectic Indium Gallium ( $\mathrm{In} / \mathrm{Ga})$, to carry out vLIBWE process on Zerodur. The light emission during the v-LIBWE process was monitored using a real-time monitoring method described in our previous works [14-16] to quickly determine the etching threshold. The etching rate and surface quality was also studied and reported.

\section{Experiment}

\subsection{Materials and Reagents}

Zerodur manufactured by Schott was obtained through a local supplier (HiLite Optronics Inc.). Zerodur slide with thickness of $1 \mathrm{~mm}+/-0.1 \mathrm{~mm}$ and double-side polishing was used for the v-LIBWE experiment. The slide was assembled with a silicone spacer and a glass slide to form a 
sealed liquid chamber. The chamber dimension is $10 \mathrm{~mm}$ wide x $10 \mathrm{~mm}$ long x $1 \mathrm{~mm}$ high. In/Ga liquid was injected into the liquid chamber using a needled (gauge \#23) syringe.

The Indium Gallium alloy is prepared according to the literature [17]. The composition of the alloy used in this study was eutectic $\mathrm{In} / \mathrm{Ga}$ (21.4 weight percent indium or 14.2 atomic percent indium). The eutectic $\mathrm{In} / \mathrm{Ga}$ is a relatively new absorber for the LIBWE process [11] and has melting point of $15.3{ }^{\circ} \mathrm{C}$ at the specific ratio of the alloy. The low melting temperature makes it convenient to handle the metallic absorber for the v-LIBWE process.

Before the etching process, the Zerodur slide was cleaned by alcohol and wiped dry using a tissue paper. After the etching, the slide was soaked in $1 \mathrm{~N} \mathrm{HCl}$ for at least 3 hours and then sonicated in $1 \mathrm{~N} \mathrm{HCl}$ for $30 \mathrm{~min}$ and then in a detergent solution, TFD4, for $30 \mathrm{~min}$. The slide was then washed by de-ionized water and blown dry by nitrogen air before surface analysis by a SEM (NOVA 200, FEI) or a laser confocal surface profiler (VK-9700 Keyence).

(a)

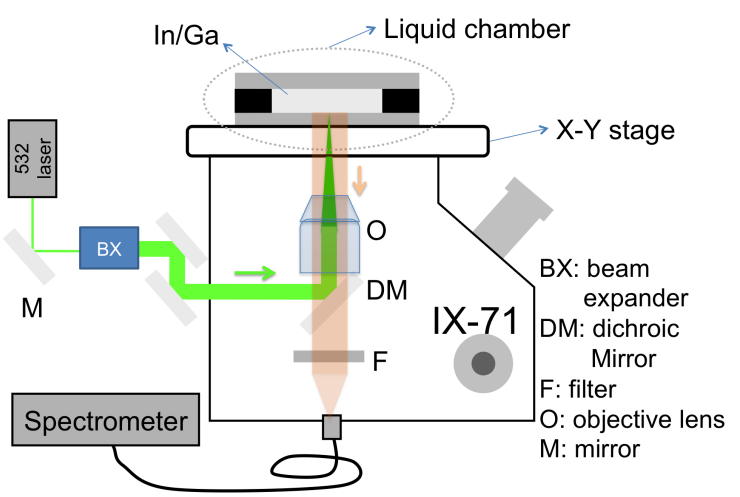

(b) Liquid chamber

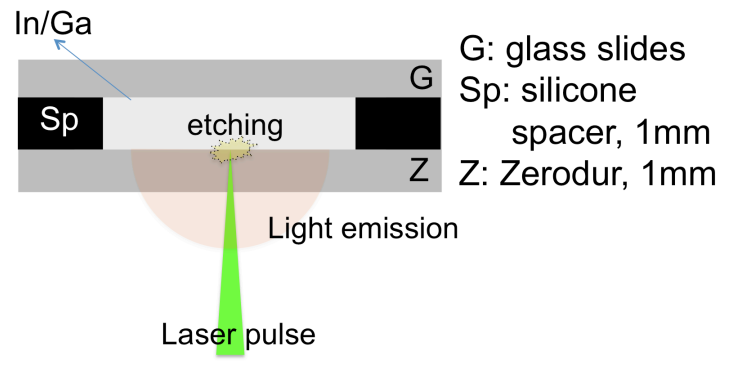

Fig. 1 (a) Optical setup for v-LIBWE and emission light measurement. (b) Liquid chamber for v-LIBWE.

\section{2 v-LIBWE setup and light emission measurement}

In this work, the emission light during the direct ablation or v-LIBWE was monitored. The emission light measurement and v-LIBWE was carried out simultaneously using the setup similar to that described in our previous work $[14,16]$. As shown in figure 1, a $532 \mathrm{~nm}$ nanosecond Qswitched laser (Ekspla, NL201, modified to output only
$532 \mathrm{~nm}$ light) was used in this study. The pulse duration is $\sim 15 \mathrm{~ns}$. The repetition rate is $2500 \mathrm{~Hz}$ unless stated otherwise. Average power of $0.4 \mathrm{~mW}$ to $35 \mathrm{~mW}$ was used in this study. The power was measured using a photodiode power meter (S120VC/PM100A, Thorlabs). Average pulse energy was calculated by dividing the average power by the repetition rate. The corresponding pulse energy was $0.16 \mu \mathrm{J}$ to $14 \mu \mathrm{J}$. The laser beam was first expanded by 10 times and then directed into an inverted microscope (Olympus, IX71). The profile of the expanded laser beam was measured by a linear CCD (Thorlabs, LC1-USB) and then fitted by Gaussian distribution function to determine the expanded beam size. The expanded beam size was $8.3 \mathrm{~mm}$.

The expanded laser beam was reflected by a notch dichroic mirror (DM, NFD-01 532, Semorock) or a 50/50 beamsplitter (SP, Omega Optical Inc.) and then focused at the first glass-liquid interface, i.e. the Zerodur-In/Ga interface, from the bottom to up by a $10 \mathrm{X}$ objective lens (Olympus Plan N 10X). When an SP is used, a long pass filter (540LP, $3^{\text {rd }}$ Millennium, Omega Optical Inc.) is used before collecting the emission light to the spectrometer. The DM, the SP and the filter provide flat spectral response in the observation range. The focused beam size $\left(1 / \mathrm{e}^{2}\right)$ was calculated by the following equation $[6,18]$ to be $2.3 \mu \mathrm{m}$.

$$
d_{0} \sim=(2 F \lambda) / d_{i} \quad \text { eq. }(1)
$$

where $d_{0}$ represents the focused beam size; $F$ is the focal length ( $=18 \mathrm{~mm}$ for objective used); $\lambda$ is the laser light wavelength, and $d_{i}$ is the initial laser beam size $(=8.3 \mathrm{~mm}$ in this study). The focused beam size, $d_{0}$, was used to calculate the laser fluence at the etching spot.

The In/Ga (i.e. the absorber) were held by the liquid chamber, as shown in figure 1(b). The laser focusing is easily achieved by observing the strong reflection of In/Ga at the Zerodur-In/Ga interface. The chamber was fixed on a computer-controlled X-Y stage. The v-LIBWE was carried out by moving the stage at $0.5 \mathrm{~mm} / \mathrm{sec}$ while the laser was focused at the Zerodur-In/Ga interface.

The emission light from the liquid chamber was analyzed by a cooled $\left(-20^{\circ} \mathrm{C}\right)$ scientific grade spectrometer (Ocean Optics QE-65000). The emission was collected by an optical fiber and delivered to the spectrometer for the analysis. The signal integration time was set as $40 \mathrm{~ms}$ to $100 \mathrm{~ms}$. The relatively long integration time ensures that the emission caused by multiple incoming laser pulses was collected and averaged.

In addition to the emission spectra, the emission intensity of some signature peaks was measured at different laser fluences in order to monitor the etching threshold.

\subsection{Trench etching by LIBWE}

The purpose of the trench etching is for measuring the etch rate per laser pulse, referred to as the per-pulse etching rate in the following. The etching threshold is the lowest laser fluence that causes the etching. 
Trenches was etched by moving the stage at $0.5 \mathrm{~mm} / \mathrm{sec}$. In order to deepen the trenches for achieving correct depth measurement, each trench was repeatedly etched for 6 beam passes. Each pass is $10 \mathrm{~mm}$ long.

The depths of the trenches were measured by the laser confocal surface profiler for shallow trenches or the SEM for deep trenches and for observing cross-sections. The per-pulse etching rate was calculated using the following equation $[5,10]$.

$$
\operatorname{Re}_{\mathrm{e} t c h}=\frac{D}{\left(d_{0} / V\right) \times n \times f} \times 1000(\mathrm{~nm} / \mathrm{pulse})
$$

, where $D$ is the etched depth; $V$ is the moving speed of the laser beam (i.e. the speed of the stage); $d_{0}$ is the focused beam size; $n$ is beam pass number, and $f$ is the laser repetition rate.

The surface quality of the Zerodur before and after etching was measured by the laser confocal surface profiler.

\section{Results and Discussion}

\subsection{Direct ablation of Zerodur}

We first tried using the $532 \mathrm{~nm}$ laser for direct ablation, i.e. without $\mathrm{In} / \mathrm{Ga}$, of the Zerodur slide. The ablation result is shown in figure 2. Apparent heat affected zone (HAZ) was observed. In addition, clear rainbow like pattern appeared in the region adjacent to the ablated region. This indicates dramatic refractive index changes, suggesting alteration of the Zerodur crystal structure in the region as far as several hundred micrometers away from the ablated line.

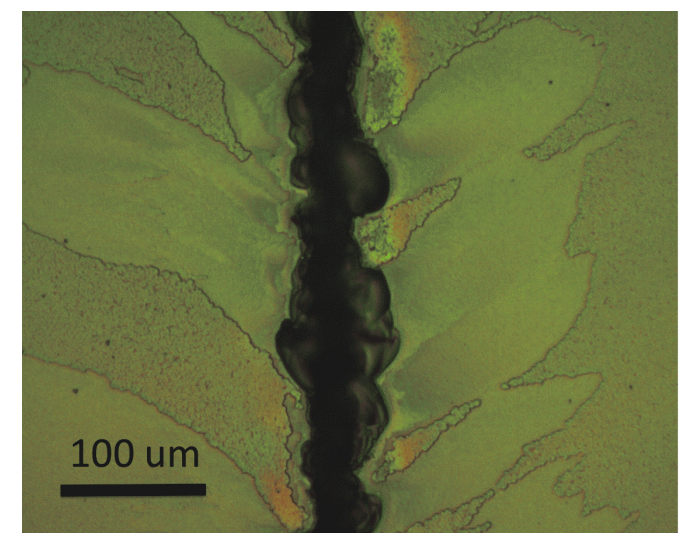

Fig. 2 A typical trench obtained by direct ablation of Zerodur by $532 \mathrm{~nm}$ laser pulse. Apparent cracking and interference pattern is observed. The laser fluence is $440 \mathrm{~J} / \mathrm{cm}^{2}$. Laser power $14 \mathrm{~mW}$. Repetition rate $1000 \mathrm{~Hz}$.
Different laser fluence was used for the ablation and the light emission was measured during the ablation. Two typical spectra are shown in figure 3 . It is clearly seen that when the ablation occurs, some sharp emission peaks were observed. Among these peaks, the $670.3 \mathrm{~nm}$ peak is the most prominent. Neither Indium nor Gallium show plasma peak close to this region[19,20]. The $670 \mathrm{~nm}$ peak is attributed to the emission of Lithium (Li) in air[21]. In our observation, the emission peak is broader than what is observed under low pressure. This broadening is caused by the atmospheric pressure[22]. The band at around $700 \mathrm{~nm}$ existed with and without ablation. This emission is probably caused by the luminescence of Zerodur and is not suitable to be used as an indication of the ablation process.

The intensity of the Li peak was measured as the laser fluence was increased. As can be seen in figure 3 and the inset, when the ablation threshold is exceeded, the peak appears and the intensity abruptly increases. In order to estimate the ablation threshold, the linear regression of the lowest three intensities that exhibited the $\mathrm{Li}$ emission peak was calculated. The regression line was extrapolated to the $\mathrm{X}$-axis and the intersection was estimated as the ablation threshold. The obtained ablation threshold is $100 \mathrm{~J} / \mathrm{cm}^{2}$. Measuring the Li emission peak is advantageous in obtaining the ablation threshold without removing the substrate from the laser and can be used for real-time monitoring of the ablation process.

Since the direct ablation does not provide crack-free surface on Zerodur, we further tried utilizing v-LIBWE for the micromachining of this material.

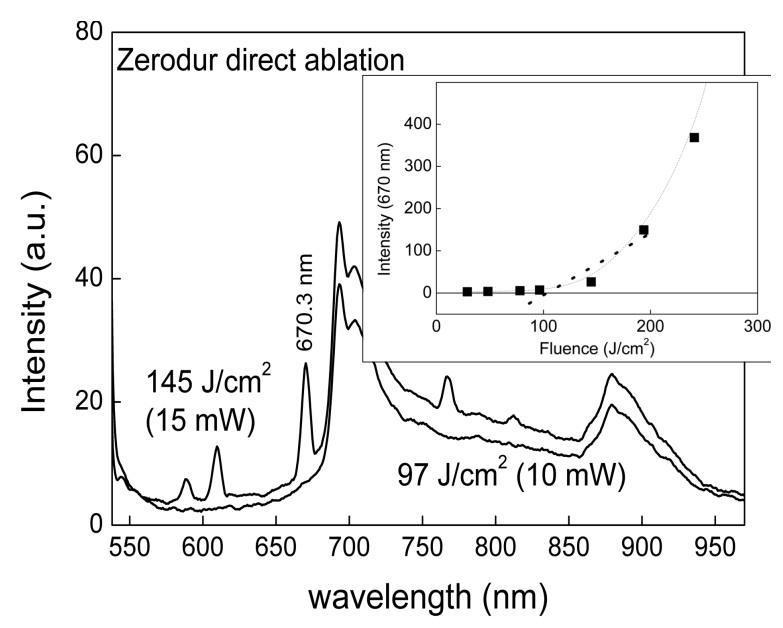

Fig. 3 Light emission spectra during the direct ablation of Zerodur. Some peaks were only observed when the laser fluence is large enough. The inset shows the abrupt intensity increase of the $670 \mathrm{~nm}$ peak when etching threshold is exceeded.

\section{2 v-LIBWE of Zerodur using In/Ga}

In addition to its low melting point, $\mathrm{In} / \mathrm{Ga}$ also has high surface tension and low viscosity[23]. These properties make it easy to handle the absorver when carrying out the v-LIBWE. In our experiment, In/Ga liquid was easily injected into and drawn out of the liquid chamber using a 
syringe manually. Moreover, crack-free trench etched using In/Ga was obtained.

A typical trench etched using the v-LIBWE is shown in figure 4 . The magnified view shows a clean and crack-free surface. The surface roughness at the bottom of the trench is $0.55 \mu \mathrm{m}$ (RMS). Although the theoretical focused beam size is $2.3 \mu \mathrm{m}$, the obtained trench width is in the range of 5 to $10 \mu \mathrm{m}$. However, at the region away from trench, no obvious distortion, cracking, or debris was observed. Although the obtained surface quality is larger than those obtained for fused silica (30 nm RMS) and sodalime (102 nm RMS) [11], this result demonstrates that good etching of Zerodur is obtained using v-LIBWE with In/Ga as the absorber. We further investigate the etching rate and etching threshold.

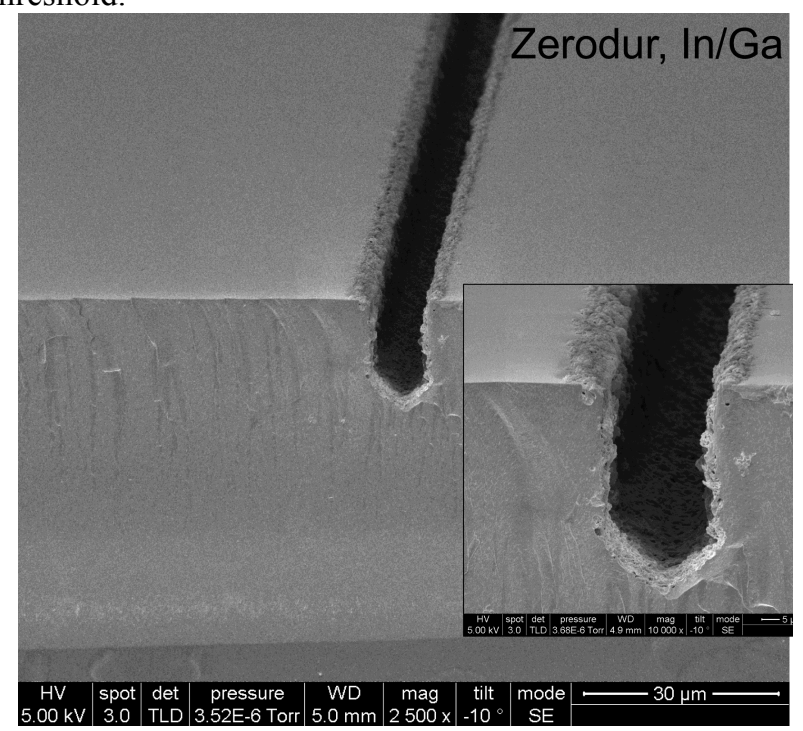

Fig. 4 SEM of a crack-free trench etched using v-LIBWE with $\mathrm{In} / \mathrm{Ga}$ as the light absorber. The laser fluence is $134 \mathrm{~J} / \mathrm{cm}^{2}$ (power $14 \mathrm{~mW}$ ). The beam pass number is 6 times. The inset shows the magnified view of the trench.

Etching rate is usually expressed as etching rate per laser pulse. It was determined by measuring the depth of a trench and then divided it with the number of laser pulses scanning through a spot. By measuring the etching rate at different laser fluence, the minimal fluence that causes the etching is referred to as the etching threshold. The result is shown in figure 5(a). The maximal etching rate shown in figure 5 is about $30 \mathrm{~nm} /$ pulse using fluence of $134 \mathrm{~J} / \mathrm{cm}^{2}$. With larger fluence than this value, cracks formation was observed in trenches undergoing repeated etching. The obtained threshold for Zerodur is $50 \mathrm{~J} / \mathrm{cm}^{2}$. This value is relatively high compared to sodalime glass $\left(0.3 \mathrm{~J} / \mathrm{cm}^{2}\right)$ using the same absorber[11].

Determining the etching threshold by measuring the etching rate is time-consuming and is an off-line method. In our previous works we have demonstrated a more efficient method that gives identical etching threshold [14-16]. As shown in figure 5(b), we measured the intensity of characteristic plasma emission, the $670 \mathrm{~nm}$ peak, at different laser fluence and then find the minimal fluence that induced the emission. The obtained etching threshold is 43 $\mathrm{J} / \mathrm{cm}^{2}$, which is a little bit lower than that obtained by the depth-measuring method. It is noticed that the v-LIBWE threshold of Zerodur is only about $1 / 2$ of that by direct ablation. For comparison, fused-silica, $\mathrm{CaF}_{2}$, and sapphire have larger difference between the direct ablation threshold and the LIBWE threshold LIBWE[4,5].

The difference in the threshold values obtained by the two methods may come from the fact that measuring the emission intensity is more sensitive in monitoring the etching process than the depth-measuring method does. As shown in figure 6 , the characteristic emission peak can be clearly observed with fluence as low as $39 \mathrm{~J} / \mathrm{cm}^{2}$, which is significantly lower than those thresholds obtained in figures 5(a) and 5(b). Since the plasma emission should be a consequence of etching process, the observation of the characteristic peak under $39 \mathrm{~J} / \mathrm{cm}^{2}$ should be an indication of the etching. We therefore conclude that the emission intensity method gives a more accurate threshold value than the depth-measuring method.

As the laser fluence increased from the threshold to about $100 \mathrm{~J} / \mathrm{cm}^{2}$, the emission intensity increased linearly, corresponding to the slow etching region between $43 \mathrm{~J} / \mathrm{cm}^{2}$ to $100 \mathrm{~J} / \mathrm{cm}^{2}$. When the fluence was larger than $100 \mathrm{~J} / \mathrm{cm}^{2}$, the slope of the intensity increase became sharper. The fluence region between $100 \mathrm{~J} / \mathrm{cm}^{2}$ to $150 \mathrm{~J} / \mathrm{cm}^{2}$ corresponds to the fast etching region. This two-region etching behavior has been observed in the LIBWE etching of several materials, such as fused silica [12,24] and sodalime glass [11].
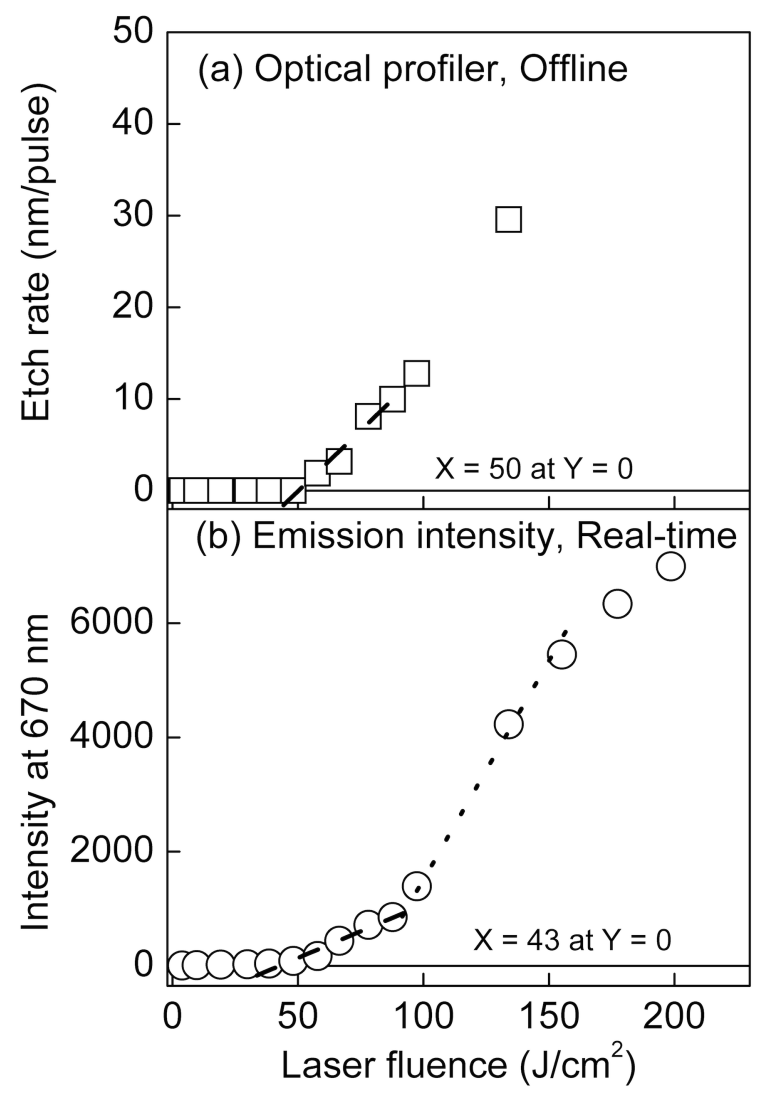

Fig. 5 Etch rate (a) and plasma light emission intensity (b) under different laser fluence. The two measurement methods give similar etching threshold but the latter one gives more detailed information on the fluence dependency of the etching behavior. 
The fluence dependency of the plasma emission showed an additional region when the fluence was larger than $\sim 150 \mathrm{~J} / \mathrm{cm}^{2}$. In this region, the slope of the emission increase became smaller than in the fast etching region. This region is not easy to be observed using the depthmeasuring approach because the crack formation in this fluence region made the depth measurement not practical. The laser fluence in this region may not be ideal for structure fabrication. However, the plasma emission in this fluence region may be helpful for the study of LIBWE mechanism under high fluence.

It is worth mention that the direct ablation threshold of Zerodur is $\sim 100 \mathrm{~J} / \mathrm{cm}^{2}$, which is inside to the crack-free fluence region of the $v$-LIBWE. This suggests that by using the fluence larger than $100 \mathrm{~J} / \mathrm{cm}^{2}$, back-side wet etching and direct ablation may occur simultaneously. This may be part of the reason why the obtained surface roughness is larger than other materials using the same absorber and the same laser source.

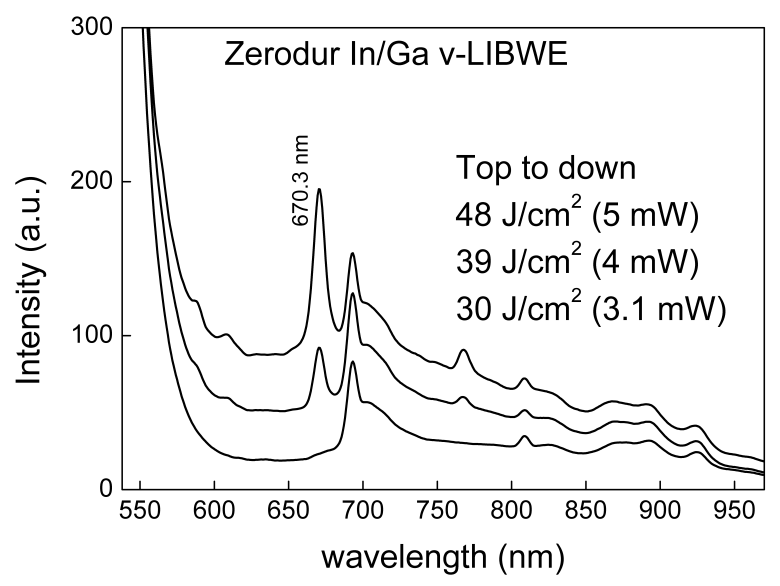

Fig. 6 Emission spectra during v-LIBWE of Zerodur at different fluences. The Li plasma emission peak $(670.3 \mathrm{~nm})$ appears when the fluence is larger than the etching threshold.

\section{References:}

[1] Schott Zerodur 2013, http://www.schott.com/advanced_optics/english/s yn/advanced_optics/products/opticalmaterials/zerodur-zero-expansion-glassceramic/zerodur/index.html, n.d.

[2] P. Hartmann, R. Jedamzik, T. Westerhoff, Zeroexpansion glass ceramic ZERODUR: recent developments reveal high potential, in: SPIE Astronomical Telescopes + Instrumentation, SPIE, 2012: pp. 845022-1-13.

[3] J. Wang, H. Niino, A. Yabe, One-step microfabrication of fused silica by laser ablation of an organic solution, Applied Physics A. 68 (1998) 111-113.

[4] J. Wang, H. Niino, A. Yabe, Micromachining of quartz crystal with excimer lasers by laserinduced backside wet etching, Applied Physics A. 69 (1999) S271-S273.

[5] R. Bohme, A. Braun, K. Zimmer, Backside etching of UV-transparent materials at the interface

\section{Conclusion}

In this work, we successfully obtained crack-free etching of Zerodur using v-LIBWE with eutectic In/Ga as the light absorber.

The plasma light emission during the ablation and the v-LIBWE process is measured and the characteristic peak of $\mathrm{Li}$ is monitored and used as an indication for the ablation and the etching process. The ablation and the etching threshold can be determined by measuring the emission intensity at different laser fluence.

We utilized two different methods to determine the etching threshold. The depth-measuring method gave a threshold of $50 \mathrm{~J} / \mathrm{cm}^{2}$ and the emission intensity method gave $43 \mathrm{~J} / \mathrm{cm}^{2}$. We argued that the latter method gave a more accurate value. In addition, the emission intensity method reveals a new fluence region in the fluence dependency of the etching.

\section{Acknowledgments:}

The support of the National Science Council Taiwan is acknowledged (Contract no. NSC 100-2113-M001-014MY3). We also thanks the financial support from the Nanoscience Prograom of Academia Sinica Taiwan.

to liquids, Applied Surface Science. 186 (2002) 276-281.

[6] J.-Y. Cheng, M.-H. Yen, C.-W. Wei, Y.-C. Chuang, T.-H. Young, Crack-free direct-writing on glass using a low-power UV laser in the manufacture of a microfluidic chip, J. Micromech. Microeng. 15 (2005) 1147-1156.

[7] C. Vass, B. Hopp, T. Smausz, F. Ignácz, Experiments and numerical calculations for the interpretation of the backside wet etching of fused silica, Thin Solid Films. 453-454 (2004) 121-126.

[8] J.-Y. Cheng, M.-H. Yen, T.-H. Young, Crackfree micromachining on glass using an economic Q-switched $532 \mathrm{~nm}$ laser, J. Micromech. Microeng. 16 (2006) 2420-2424.

[9] J.-Y. Cheng, M.-H. Yen, T.-H. Young, Crackfree micromachining on glass using an economic nanosecond green laser for manufacturing microfluidic chip, in: Kyoto Japan, 2006.

[10] J.-Y. Cheng, M.-H. Yen, W.-C. Hsu, J.-H. Jhang, T.-H. Young, ITO patterning by a low power Qswitched green laser and its use in the fabrication of a transparent flow meter, J. Micromech. Microeng. 17 (2007) 2316-2323.

[11] M.-H. Yen, C.-W. Huang, W.-C. Hsu, T.-H. Young, K. Zimmer, J.-Y. Cheng, Crack-free mi- 
cromachining on glass substrates by visible LIBWE using liquid metallic absorbers, Applied Surface Science. 257 (2010) 87-92.

[12] C. Vass, B. Kiss, J. Kopniczky, B. Hopp, Applied Surface Science, Applied Surface Science. 278 (2013) 241-244.

[13] K. Zimmer, R. Bohme, Laser-Induced Backside Wet Etching of Transparent Materials with Organic and Metallic Absorbers, Laser Chemistry. 2008 (2008) 1-13.

[14] Ji-Yen Cheng, Mansoureh Z Mousavi, ChunYing Wu, Hsieh-Fu Tsai, Blue Light Plasma Emission During LIBWE Using $532 \mathrm{~nm}$ Qswitched Nanosecond Laser, JLMN-Journal of Laser Micro/Nanoengineering. 7 (2012) 87-92.

[15] J.-Y. Cheng, M.S.Z. Mousavi, C.-Y. Wu, H.-F. Tsai, Plasma emission from glass/liquid interface for real-time monitoring of LIBWE process, in: Takamatsu, Japan, 2011.

[16] J.-Y. Cheng, M.Z. Mousavi, C.-Y. Wu, H.-F. Tsai, Blue light emission from glass/liquid interface for real-time monitoring of laser-induced etching process, J. Micromech. Microeng. 21 (2011) 075019.

[17] J.N. Koster, Directional Solidification and Melting of Eutectic GaIn, Cryst. Res. Technol. 34 (1999) 1129-1140.

[18] A.E. Siegman, Lasers, University Science Books, Sausalito, CA, 1986.

[19] A.K. Shuibov, L.L. Shimon, A.J. Dashchenko, M.P. Chuchman, Optical Characteristics of Erosion Gallium, Indium and Copper Laser Plasma, (2000) 1-5.

[20] A.C. Popescu, S. Beldjilali, G. Socol, V. Craciun, I.N. Mihailescu, J. Hermann, Analysis of indium zinc oxide thin films by laser-induced breakdown spectroscopy, J. Appl. Phys. 110 (2011) 083116.

[21] H. Kurniawan, S. Nakajima, J.E. Batubara, M. Marpaung, M. Okamoto, K. Kagawa, Laserinduced shock wave plasma in glass and its application to elemental analysis, Applied Spectroscopy. 49 (1995) 1067-1072.

[22] O.A. Bukin, I.V. Bazarov, N.V. Bodin, V.I. Tsarev, A.Y. Maior, E.N. Bol'shakova, Mechanisms of broadening of emission lines of a laserinduced plasma generated on the surface of rigid targets, Journal of Applied Spectroscopy. 67 (2000) 320-326.

[23] M.D. Dickey, R.C. Chiechi, R.J. Larsen, E.A. Weiss, D.A. Weitz, G.M. Whitesides, Eutectic Gallium-Indium (EGaIn): A Liquid Metal Alloy for the Formation of Stable Structures in Microchannels at Room Temperature, Adv. Funct. Mater. 18 (2008) 1097-1104.

[24] T. Sato, Y. Kawaguchi, R. Kurosaki, A. Narazaki, W. Watanabe, H. Niino, Laser-Induced Backside Wet Etching Employing Green DPSS Laser and Liquid Metallic Absorber, JLMN-Journal of Laser Micro/Nanoengineering. 6 (2011) 204-208. 